Fecha de recepción: febrero 2008 Fecha de aceptación: marzo 2008 Versión final: diciembre 2008

\section{Las pymes y la función de la comunicación}

Patricia Iurcovich ${ }^{*}$

(*) Licenciada en Publicidad. Master en Comunicación Institucional. Consultora en Comunicación Corporativa, especializada en el campo de las Comunicaciones Integradas. Profesora en el MBA de la Escuela de Economía y Negocios (Universidad de Belgrano) en el campo de las Comunicaciones de Marketing. Profesora de la Carrera Relaciones Públicas de la Facultad de Diseño y Comunicación de la Universidad de Palermo, en Imagen Corporativa. Profesora de la Fundación Hillel.

Resumen: La complejidad de los mercados actuales y la dinámica de los cambios definen un escenario para las Pymes en el que la función de comunicación se constituye en un rol clave, definiendo una serie de variables muy específicas, desafiantes para los profesionales de las comunicaciones de la época actual, cuya gestión está impregnada por la complejidad y la capacidad de gestionar de manera planificada y metodológica, generando una ventaja competitiva, en un marco dinámico y cambiante característico de esta tipología estructural. En este contexto, las PyMEs se comienzan a levantar como posibles promotoras de la producción industrial y de servicios, al mismo tiempo que se constituyen en una interesante posibilidad de especialización para los profesionales de las Relaciones Públicas, quienes tienen las herramientas necesarias para recuperar su identidad, revalorizar su cultura organizacional como base de la innovación y contribuir desde la gestión de la comunicación a la creación de valor agregado.

Palabras claves: Relaciones Públicas - Comunicación de Pymes - Comunicaciones Integradas Pymes - estrategias - competitividad - organización - identidad - cultura organizacional - gestión comunicacional - planes de comunicación - comunicación empresaria - comunicación institucional.

[Resúmenes en inglés y portugués en las páginas 96-97]

El desafío de la época actual para los profesionales de las comunicaciones es realizar su gestión con toda la complejidad y la velocidad de los cambios. Tal es la velocidad, que se corre el riesgo de quedar marginados del cambio o de convertirse rápidamente en personas u organizaciones obsoletas. Para evitar este efecto, la capacidad de adaptación debe ser más dinámica, y esto se convierte a la vez en un tremendo peso individual y organizacional. En este contexto, surge con mayor fuerza cada día, el rol clave de la función de la comunicación en las Pequeñas y Medianas Empresas (PyMes).

En un mercado competitivo, complejo, con crisis y dinámicas como las que se vienen observando y son protagonistas de esta última década, la mayoría de la empresas cuentan con estrategias, correctas $o$ incorrectas, pero estrategias al fin. Esto supone competir. En este marco de empresas se encuentran no sólo las grandes corporaciones sino las pequeñas y medianas empresas. Y como dice Alberto Levy, no hay Pymes, hay empresas, pequeñas, medianas, grandes. 
Entonces, la ventaja competitiva surge de tener un plan y una metodología superior a la de la competencia y un equipo humano altamente capacitado.

Pero este diagnóstico y la modernización de los métodos de gestión como llave del crecimiento y desarrollo de las organizaciones constituyen una verdad sobre la que no se necesita hacer notar. Es una forma de afirmar lo obvio: quien no se contacta con el medio exterior tiende a su desaparición.

En la actualidad muchas PyMEs han podido encontrar en estos nuevos escenarios una salida: reconvertirse según el contexto y ofertar según la nueva demanda. Lo pudieron hacer porque llevaron a cabo las gestiones necesarias utilizando su capacidad de acción.

Las empresas que han avizorado la oportunidad -y que habían modernizado su línea de producción y sus cuadros gerenciales- entraron al nuevo escenario con fuerza y protagonismo. Poder sostener la marcha, crecer y afianzarse, es un trabajo de largo plazo que merece ciertas consideraciones. Pero también estas empresas sirven como modelos a copiar por otras PyMEs.

\section{El contexto de gestión}

Cuando se repasa la bibliografía de diagnóstico y gestión de las PyMEs, se encuentra con que antes de la década del ' 90 , estas empresas eran sinónimo de atraso, económicamente pequeñas para constituir una fuerza en el PBI y por supuesto con un sistema de producción que no constituía ninguna pauta de calidad.

Hoy, habiendo superado la década de la convertibilidad, las PyMEs se comienzan a levantar como posibles promotoras de la producción industrial y de servicios, tal como sucede en otras partes del mundo.

La gran empresa ha entrado en crisis porque ha entrado en crisis todo lo que expresa uniformidad y ritmos autoritariamente establecidos, jerarquías rígidas y encuadres rudamente normativos.

Cuando el desafío se expresa en necesidades de flexibilidad, descentralización, desburocratización, liderazgos horizontales, espacios compartidos, agilidad y adaptaciones rápidas, personalización de la gestión y del consumo, artesanía y trabajos a medida o de series reducidas; las PYMES emergen con una identidad clara, natural, más consonante a todos estos conceptos que la gran empresa, quien estimula, desde su gigantismo, una reconversión exógena para las pequeñas y medianas empresas. El desafío de las pequeñas y medianas empresas está en la recuperación de una identidad, en la revalorización de su cultura organizacional como base de la innovación. Estas empresas tienen ventajas competitivas desde lo cultural: confianza en el éxito, personalismos inquebrantables ante los vaivenes económicos, métodos artesanales de gestión, etc.

\section{¿Cuál es la contribución de la comunicación en las Pymes?}

La comunicación tiene un rol preponderante en las empresas tanto a nivel interno como externo. Primeramente, el objetivo de la comunicación es construir el concepto de identidad, eligiendo aquellos atributos pertinentes a la organización, que la distingan como tal. Definiendo la misión y visión de la empresa, el posicionamiento frente al cliente y al resto de sus públicos, la política con los empleados, etc.

Todas estas son acciones y funciones que las herramientas de las comunicaciones integradas - de las Relaciones Públicas, el marketing, la publicidad y la comunicación organizacional- pueden potenciar, haciendo más eficaces y eficientes los resultados. 
Es relativamente cierto que estas herramientas en muchos casos son consideradas sólo para las grandes empresas, ¿las razones?, muchas. Entre ellas la percepción que se tiene por parte de algunos empresarios de las Pymes, que entienden a la comunicación como gasto y no como inversión. Además, la adecuación gerencial de estas empresas a los nuevos escenarios. La competencia, las acciones de marketing, el salir al exterior, el participar en ferias internacionales y nacionales presupone que la empresa necesita aggiornarse en términos de herramientas de comunicación.

Acciones de CRM (Gerenciamiento de la Relación con el Cliente), el diseño y desarrollo de la papelería comercial, la necesidad de construcción de una marca, el comunicarla, la relación con los empleados, la capacitación, la realización de una web, son sólo algunas de las contribuciones que la comunicación aporta a las organizaciones.

Hasta hace unos años la comunicación integrada era terreno de las grandes empresas, hoy si una empresa quiere crecer, competir, ser responsable con la sociedad, con los empleados, con el mundo debe entonces incluir a la comunicación en su presupuesto.

$\mathrm{Si}$ las pequeñas y medianas empresas pudieran crear valor agregado a sus organizaciones y a sus productos: como el valor marca, el packaging con un diseño estético y/o reforzar sus vínculos con sus públicos internos y externos, podrían generar mecanismos aún más profundos tendiendo lazos con diversos sectores de la sociedad, entre ellos con el Estado y el sector privado e internacional facilitando a través de una buena estructura comunicacional su desarrollo.

Es bien sabido que no es sencillo aplicar una gestión comunicacional en estas empresas. En principio, porque la venta de modelos de gestión apelando al éxito logrado en contextos organizacionales de gran alcance, no contiene garantías de éxito. Por otro lado, los altos costos que ello implica, la poca inversión, la reticencia a iniciar planes de reingeniería por parte del Estado para con éstas, el contexto de incertidumbre económica y las particularidades organizacionales/culturales con que cuentan, no resulta del todo viable la incorporación de las comunicaciones integradas. Pero, también es bien sabido que: para dar un paso hay que tener decisión, para dar 100 pasos hay que dar primero uno.

No se trata de encontrar las diferenciaciones especulativas, sino de encontrar las articulaciones que eludan las superposiciones de roles, tareas y funciones, y de transformar los conocimientos de los comunicadores en acciones concretas y funcionales a un servicio, que en las PyMEs será la mejora continua del negocio.

\section{Cómo contribuye el consultor en la gestión de una buena comunicación}

Cuando se trata de elegir un asesoramiento externo en el campo de la comunicación, lo primero que habría que observar frente a una Pyme es a cargo de quién se encuentra, quiénes son sus responsables, qué cultura manejan a nivel Organización, si creen que la comunicación es verdaderamente el camino adecuado para hacer conocer sus productos y/o servicios. Si creen en la comunicación como valor agregado.

Recién a partir de esta premisa es que el consultor puede avanzar en algún tipo de rumbo estratégico del negocio y de la comunicación. Para ello se deben describir algunos aspectos relacionados con el ejercicio de la creatividad que deberían desarrollar los directivos y/o empleados. Pensar inicialmente en crear una cultura propia, diferente de la heredada, (más si se trata de empresas familiares).

Pensar en capacitar al personal, es básico. Las Pymes generalmente hacen foco en la producción y no tanto en los componentes más blandos como es la cultura, la capacitación, el desarrollo de la visión, valores, identidad de la misma y comunicación. Es casi como si observásemos un cuerpo humano, 
el hemisferio derecho armado por variables más duras (producto, servicio, aspectos tangibles), y el hemisferio izquierdo con un contenido más versátil, en donde aspectos como la cultura, los valores, no se cambian día a día sino que por el contrario deben sustentarse, deben constituirse en los pilares sobre los cuales los empleados forjen su camino.

Para todo ello es importante que la capacitación exista, no sólo a nivel producto-servicio sino como eje para que la comunicación hacia fuera sea clara y se traduzca en una buena atención, en lineamientos claros con los clientes, en devolución de llamados y correos electrónicos, en calidad de atención. Todo comienza por los directivos, todo comienza por la cultura que se quiere crear, sin ello no hay labor del consultor alguna. El consultor necesita apoyo para poder insertarse en la Organización y trabajar en conjunto con el líder, al igual que en una empresa más grande. Y esto no tiene que ver únicamente con un tema financiero sino con la decisión de pasar de una oficina, de un emprendimiento, a una empresa. Las Pymes son empresas y deben comportarse como tales.

Las Pymes pierden tiempo y dinero emparchando errores por no planificar, creo que ésta es la base de los temas que giran en torno a este artículo. No hay comunicación sin planificación, con lo cual si estamos hablando de la función de la comunicación en las Pymes, deberíamos cuestionarnos hacia dónde vamos, si queremos crecer como empresa.

Es interesante cuando uno recorre el camino de los emprendimientos ver que lo primero que apareció en los pequeños o no tan pequeños emprendimientos es la visión del negocio, la posibilidad de ver más allá, de imaginarse aquello que consideramos propio, armado por nosotros mismos, con una idea de futuro.

Cuando Bill Gates pensó en lo que sería hoy por hoy Microsoft, no creo que se haya imaginado que llegaría a este punto, pero creyó en su idea y no se apartó de ella; algo similar debería ocurrir con las Pymes; lógico que es más fácil crear desde cero un modelo de cultura propia, que reorientar la cultura heredada, más si es generacionalmente heredada.

Los consultores podemos apoyar, guiar, delinear, acompañar, casi como un líder lo hace en su Organización, pero la primer pregunta que debemos hacernos a nosotros mismos y a quienes tengamos enfrente es ¿hacia dónde vamos?, sólo desde allí podremos pararnos y relevar la industria, el sector, ver con qué recursos contamos, financieros, tecnológicos, humanos y porqué no, comunicacionales. Es desde el orden que podemos partir, no desde arreglar cada situación por separado. Si se entiende así la comunicación se podrá capacitar internamente a los empleados y aplicar las herramientas de acción más adecuadas (publicidad, CRM, etc). De lo contrario eligiendo sólo herramientas externas, las Pymes mantendrán siempre un abismo entre sus empleados puertas adentro y el mundo; ellos pueden acceder a muchas cosas que el número uno ni se imagina (descuentos con comercios, alianzas, beneficios en la capacitación). Solo así crearán una empresa fuerte, innovadora, como tantas que ya existen, en donde la camiseta del empleado sea la mejor herramienta elegida. Si es así y se comprueba, todas las demás funcionarán.

Summary: The complexity of the present markets and the dynamics of the changes define a scenario for Small Companies in which the communication function is constituted in a key roll, defining specific challenging variables for communication professionals, whose management is impregnated by the complexity and the capacity to manage in a planned and methodological way, generating a competitive advantage, in a dynamic and changing frame which is characteristic of this organizational structure. In this context Small Companies are begun to raise like possible promoters of industrial production of both products and services, and at the same time they are constituted in an interesting option for public 
relations professionals specialization, that have the tools needed to help them to recover their identity, to revalue its corporate culture as it bases of the innovation and to contribute from the management of communication to the generation of added value.

Key words: Communication in Small Companies - communication management - communication plans - competitiveness - corporate communication - corporate culture - identity - Integrated Communications - organization - Public relations - small companies - strategies.

Resumo: A complexidade dos mercados actuàis e a dinâmica das mudanças definem um cenário para as MPEs onde a comunicação e um ponto chave, definindo uma serie de variáveis muito especificas, desafiantes para os profissionais das comunicações da atualidade, cuja gestão esta impregnada pela complexidade e a capacidade de gestão planejada, metodológica e gerando uma vantagem competitiva, num marco dinâmico e cambiante característico desta tipologia estrutural. Neste contexto as MPEs comenzan a se levantar como possíveis promotoras da produção industrial e de serviços, ao tempo que constituem-se numa interessante possibilidade de especialização para os profissionais das relações publicas, os quais tem as ferramentas necessárias para sua identidade, revalorizar sua cultura organizativa como base da inovação e contribuir desde a gestão da comunicação a criação de valor agregado.

Palavras chave: competitividade - Comunicação de MPEs - Comunicaçãos integradas - cultura organizativa - estratégias - gestão comunicacional - identidade - MPEs - organização - Relações públicas. 